Article

\title{
Transfer Learning with Deep Convolutional Neural Network (CNN) for Pneumonia Detection Using Chest X-ray
}

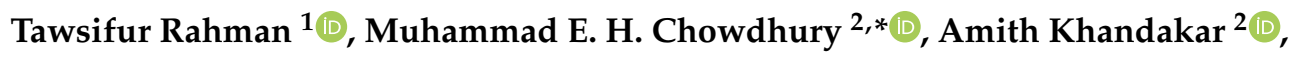 \\ Khandaker R. Islam ${ }^{3}$, Khandaker F. Islam ${ }^{2}$, Zaid B. Mahbub ${ }^{4}$, Muhammad A. Kadir ${ }^{1}$ (D) \\ and Saad Kashem ${ }^{5}$ \\ 1 Department of Biomedical Physics \& Technology, University of Dhaka, Dhaka 1000, Bangladesh; \\ tawsifurrahman@bmpt.du.ac.bd (T.R.); kadir@du.ac.bd (M.A.K.) \\ 2 Department of Electrical Engineering, Qatar University, Doha 2713, Qatar; amitk@qu.edu.qa (A.K.); \\ khlipa@gmail.com (K.F.I.) \\ 3 Department of Orthodontics, Bangabandhu Sheikh Mujib Medical University, Dhaka 1000, Bangladesh; \\ khreaj@gmail.com \\ 4 Department of Mathematics and Physics, North South University, Dhaka 1229, Bangladesh; \\ zaid.mahbub@northsouth.edu \\ 5 Faculty of Robotics and Advanced Computing, Qatar Armed Forces-Academic Bridge Program, \\ Qatar Foundation, Doha 24404, Qatar; skashem@qf.org.qa \\ * Correspondence: mchowdhury@qu.edu.qa; Tel.: +974-31010775
}

Received: 4 March 2020; Accepted: 8 April 2020; Published: 6 May 2020

check for updates

\begin{abstract}
Pneumonia is a life-threatening disease, which occurs in the lungs caused by either bacterial or viral infection. It can be life-endangering if not acted upon at the right time and thus the early diagnosis of pneumonia is vital. The paper aims to automatically detect bacterial and viral pneumonia using digital x-ray images. It provides a detailed report on advances in accurate detection of pneumonia and then presents the methodology adopted by the authors. Four different pre-trained deep Convolutional Neural Network (CNN): AlexNet, ResNet18, DenseNet201, and SqueezeNet were used for transfer learning. A total of 5247 chest X-ray images consisting of bacterial, viral, and normal chest $\mathrm{x}$-rays images were preprocessed and trained for the transfer learning-based classification task. In this study, the authors have reported three schemes of classifications: normal vs. pneumonia, bacterial vs. viral pneumonia, and normal, bacterial, and viral pneumonia. The classification accuracy of normal and pneumonia images, bacterial and viral pneumonia images, and normal, bacterial, and viral pneumonia were $98 \%, 95 \%$, and $93.3 \%$, respectively. This is the highest accuracy, in any scheme, of the accuracies reported in the literature. Therefore, the proposed study can be useful in more quickly diagnosing pneumonia by the radiologist and can help in the fast airport screening of pneumonia patients.
\end{abstract}

Keywords: pneumonia; bacterial and viral pneumonia; chest X-ray; deep learning; transfer learning; image processing

\section{Introduction}

Pneumonia is considered the greatest cause of child fatalities all over the world. Approximately 1.4 million children die of pneumonia every year, which is $18 \%$ of the total children died at less than five years old [1]. Globally, overall, two billion people are suffering from pneumonia every year [1]. Pneumonia is a lung infection, which can be caused by either bacteria or viruses. Luckily, this bacterial or viral infectious disease can be well treated by antibiotics and antivirals drugs. 
Nevertheless, faster diagnosis of viral or bacterial pneumonia and the consequent application of correct medication can help significantly to prevent deterioration of a patient's condition, which eventually leads to death [2]. Chest X-rays are currently the best method for diagnosing pneumonia [3]. X-ray images of pneumonia are not very clear and are often misclassified to other diseases or other benign abnormalities. Moreover, the bacterial or viral pneumonia images are sometimes miss-classified by the experts, which leads to wrong medication being given to the patients and thereby worsening the condition of the patients [4-6]. There are considerable subjective inconsistencies in the decisions of radiologists reported in diagnosing pneumonia. There is also a lack of trained radiologists in low resource countries (LRC), especially in rural areas. Therefore, there is a pressing need for computer-aided diagnosis (CAD) systems, which can help the radiologists in detecting different types of pneumonia from the chest X-ray images immediately after the acquisition.

Currently, many biomedical complications (e.g., brain tumor detection, breast cancer detection, etc.) are using Artificial Intelligence (AI)-based solutions [7-10]. Among the deep learning techniques, convolutional neural networks (CNNs) have shown great promise in image classification and therefore widely adopted by the research community [11]. Deep Learning Machine learning techniques on chest X-rays are gaining popularity as they can be easily used with low-cost imaging techniques and there is an abundance of data available for training different machine-learning models. Several research groups [1,12-25] have reported the use of deep machine learning algorithms in the detection of pneumonia; however, only one article [16] has reported the classification of bacterial and viral pneumonia.

There are many works where the authors tried varying the parameters of deep layered CNN for pneumonia detection. The pattern of diffuse opacification in the lung radiograph due to pneumonia can be alveolar or interstitial. Patients with alveolar infiltration on the chest radiograph, especially those with lobar infiltrates, have laboratory evidence of a bacterial infection [26]. Again, interstitial infiltrations on radiograph may be linked with viral pneumonia [27]. These might be the distinctive features in the machine learning algorithms in differentiating viral and bacterial pneumonia. Some researchers have promising results, such as Liang et al. [18], Vikash et al. [28], Krishnan et al. [29], and Xianghong et al. [30]. Some groups have combined CNN with rib suppression and lung filed segmentation for other classification tasks, while some highlighted a visualization technique in CNN to find the region of interest (ROI) that can be used to identify pneumonia and distinguish between bacterial and viral types in pediatric chest $\mathrm{X}$-rays. The concept of transfer learning in deep learning framework was used by Vikash et al. [28] for the detection of pneumonia using pre-trained ImageNet models [31] and their ensembles. A customized VGG16 model was used in Xianghong et al. [30], which consists of two parts, lung region identification with a fully convolutional network (FCN) model and pneumonia category classification using a deep convolutional neural network (DCNN). A dataset containing 32,717 patients' $\mathrm{X}$-rays were used in a deep learning technique by Wang et al. [12] producing promising results. In [13] the authors further went on using data augmentation along with CNN to get better results by training on a small set of images. Rajpurkar et al. [32] reported a 121-layer CNN on chest $\mathrm{X}$-rays to detect 14 different pathologies, including pneumonia using an ensemble of different networks. Jung et al. [33] used a 3D Deep CNN with shortcuts and dense connections which help in solving the gradient vanishing problem. Jaiswal et al. [17] and Siraz et al. [34] used a deep neural network called Mask-RCNN (Region based Convolutional Neural Network) for the segmentation of pulmonary images. This was used along with image augmentation for pneumonia identification, which was ensembled with RetinaNet to localize pneumonia. A pretrained DenseNet-121 and feature extraction techniques were used in the accurate identification of 14 thoracic diseases in [16]. Sundaram et al. [35] used AlexNet and GoogLeNet [36] with data augmentation to obtain an Area Under the Curve (AUC) of 0.94-0.95. The same authors had better results of AUC of 0.99 using modified two-network ensemble architecture.

The highest accuracy reported in the above-mentioned literatures in classifying the normal vs. pneumonia patients and bacterial vs. viral pneumonia using X-ray images using deep learning algorithms was $96.84 \%$ and $93.6 \%$, respectively. Therefore, there is significant room for improving 
the result either by using different deep learning algorithms or modifying the existing outperforming algorithms or combining several outperforming algorithms as an ensemble model to produce a better classification accuracy, particularly in classifying viral and bacterial pneumonia. The proposed study is reporting a transfer learning approach using four different pre-trained network architectures (AlexNet, ResNet18, DenseNet201, and SqueezeNet) and analyzed their performances. The key contribution of this work is to provide a CNN-based transfer-learning approach using different pre-trained models to detect pneumonia and classify bacterial and viral pneumonia with higher accuracy compared to the recent works. In addition, the paper also provides the methodological details of the work, which can be utilized by any research group to take the benefit of this work. Moreover, radiographic findings are poor indicators for the diagnosis of the cause of pneumonia until now. Therefore, the motivation of the present study was to utilize the power of machine learning, firstly to diagnose pneumonia by analyzing radiograph and secondly to differentiate viral and bacterial pneumonia with better accuracy.

The rest of the paper is divided in the following section: Section 2 summarizes different pre-trained networks used for this study and Section 3 describes the methodology used in the study, where the details of the dataset and the pre-processing steps to prepare the data for training and testing are provided. Section 4 provides the results of the classification algorithms, which is compared with some other recent studies, and the results are discussed in this section. Finally, the conclusion is presented in Section 5 .

\section{Background of Deep Machine Learning Algorithms}

\subsection{Convolutional Neural Networks (CNNs)}

As discussed earlier, CNNs have been popular due to their improved performance in image classification. The convolutional layers in the network along with filters help in extracting the spatial and temporal features in an image. The layers have a weight-sharing technique, which helps in reducing computation efforts $[37,38]$.

Architecture wise, CNNs are simply feedforward artificial neural networks (ANNs) with two constraints: neurons in the same filter are only connected to local patches of the image to preserve spatial structure and their weights are shared to reduce the total number of the model's parameters. A CNN consists of three building blocks: (i) a convolution layer to learn features, (ii) a max-pooling (subsampling) layer is to down sample the image and reduce the dimensionality and thereby reduction in computational efforts, and (iii) a fully connected layer to equip the network with classification capabilities [39]. The architectural overview of CNN is illustrated in Figure 1.

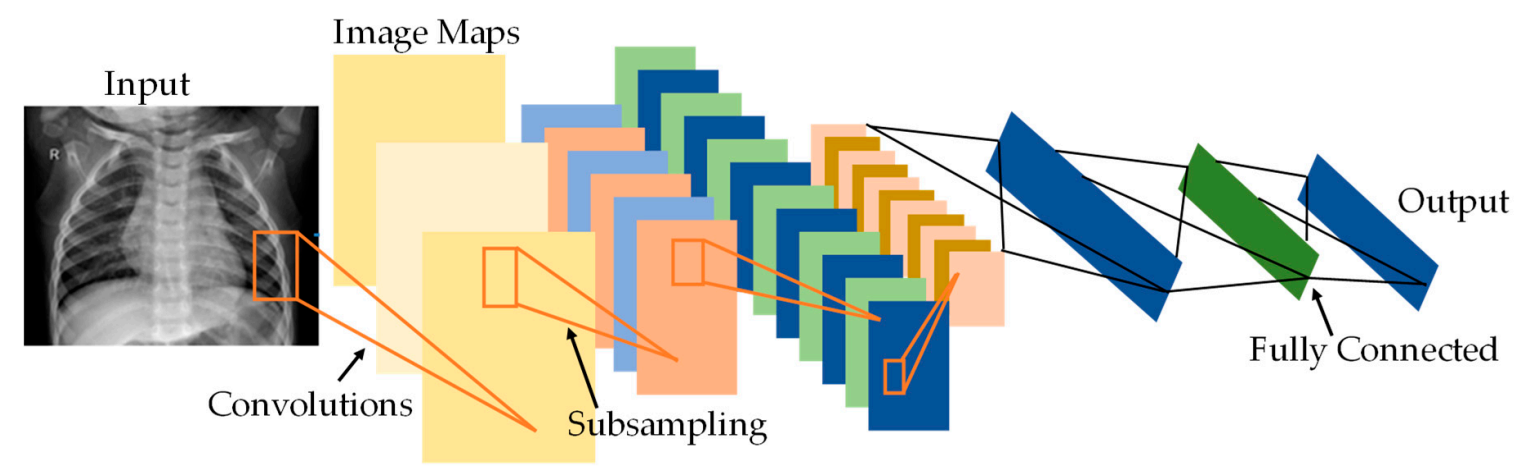

Figure 1. Convolutional Neural Network (CNN) Architecture.

\subsection{Deep Transfer Learning}

CNNs typically outperform in a larger dataset than a smaller one. Transfer learning can be useful in those applications of $\mathrm{CNN}$ where the dataset is not large. The concept of transfer learning is shown 
in Figure 2, where the trained model from large datasets, such as ImageNet [40,41], can be used for application with a comparatively smaller dataset.

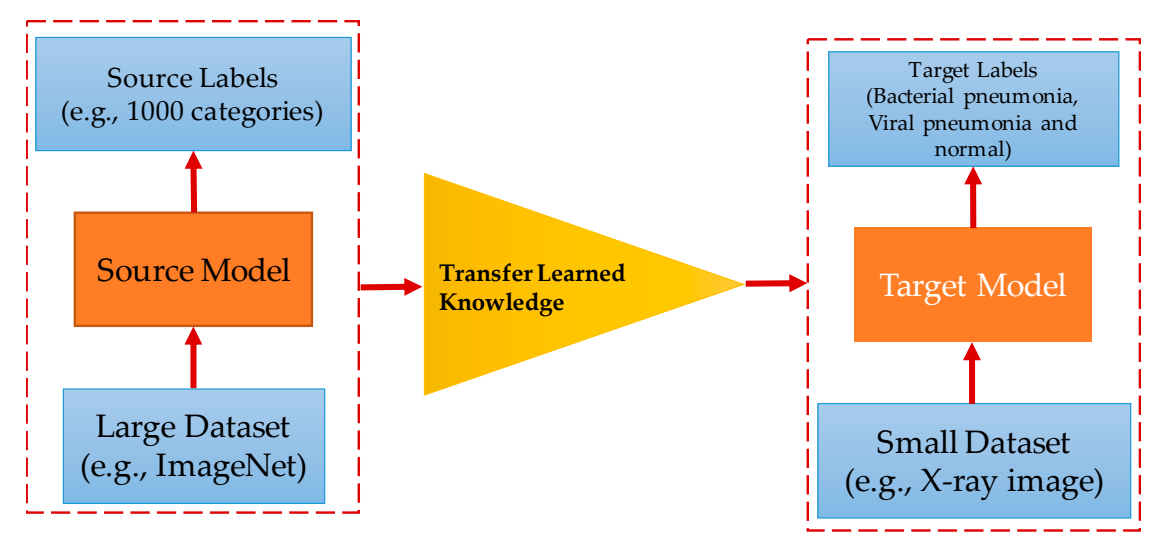

Figure 2. Concept of transfer learning.

Recently, transfer learning has been successfully used in various field applications, such as manufacturing, medical and baggage screening [42-44]. This removes the requirement of having large dataset and also reduces the long training period, as is required by the deep learning algorithm when developed from scratch $[45,46]$.

\subsection{Pre-Trained Convolutional Neural Networks}

In this study, four well-known pre-trained deep learning CNNs: AlexNet [11], ResNet [47], DenseNet [47], and SqueezeNet [48] were used for pneumonia detection. A brief introduction of these pre-trained networks is mentioned below:

\subsubsection{AlexNet}

AlexNet can classify more than 1000 different classes using deep layers consisting of 650k neurons and 60 million parameters. The network is made up of five convolutional layers (CLs) with three pooling layers, two fully connected layer (FLCs), and a Softmax layer [11]. The dimension of input image for the AlexNet has to be $227 \times 227 \times 3$ and the first CL converts input image with 96 kernels sized at $11 \times 11 \times 3$ having a stride of four pixels, which is the input to the second layer [49], and the remaining details are summarized in Figure 3.

\subsubsection{ResNet18}

ResNet, which is a short form of Residual Network (Figure 4), was originally developed to two problems, such as the vanishing gradient and degradation problem [47]. Residual learning tries to solve both these problems. ResNet has three different variants: ResNet18, ResNet50, and ResNet101 based on the number of layers in the residual network. ResNet was successfully used in biomedical image classification [48] for transfer learning. In this paper, we have used ResNet18 for the pneumonia detection. Typically, deep neural network layers learn low- or high-level features during training, while ResNet learns residuals instead of features. 


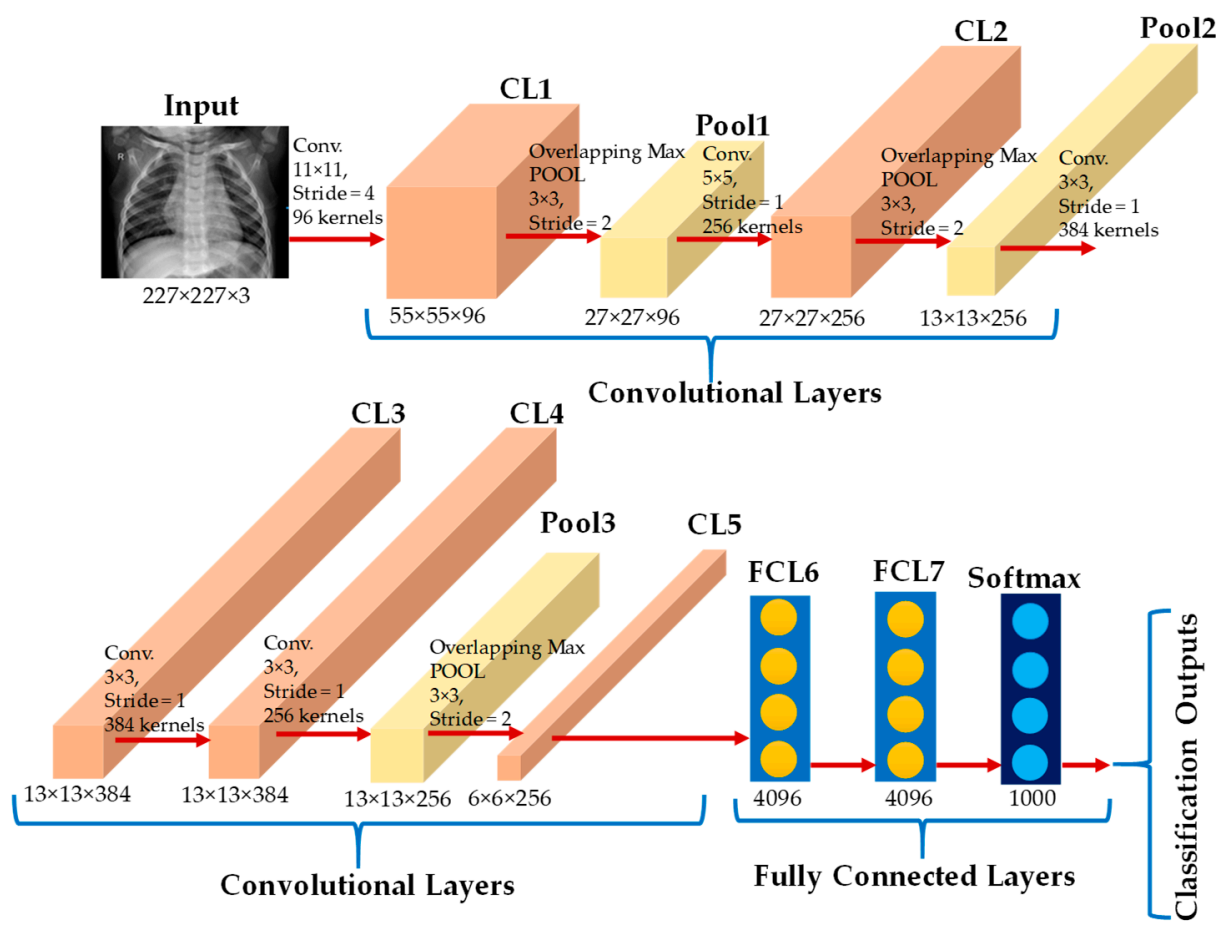

Figure 3. AlexNet Structure.

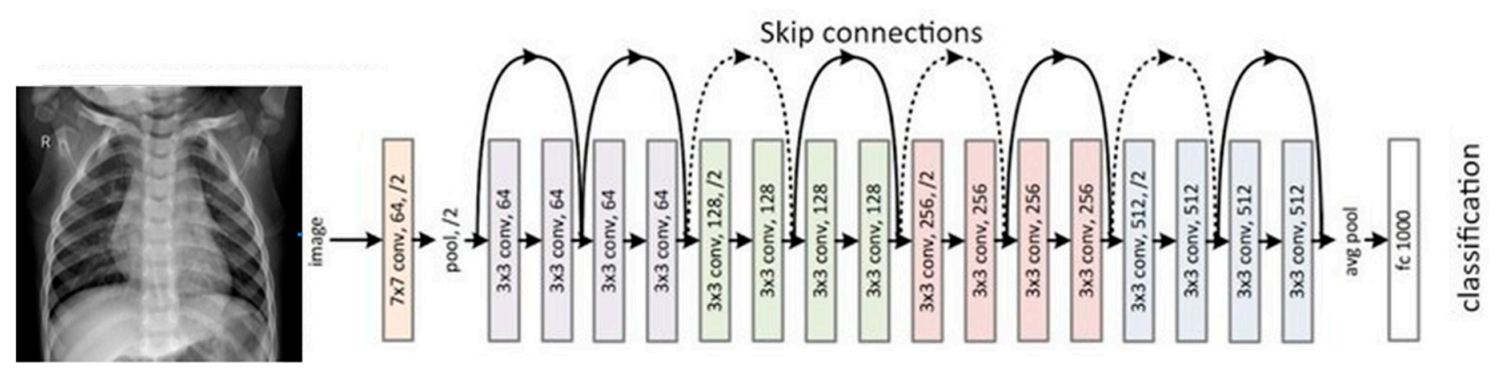

Figure 4. Structure of ResNet18.

\subsubsection{DenseNet201}

DenseNet, which is a short form of the Dense Convolutional Network, needs less numbers of parameters than a conventional CNN, as it does not learn redundant feature maps [50]. The layers in DenseNet are very narrow, i.e., 12 filters, which add a lesser set of new feature-maps. DenseNet has four different variants: DenseNet121, DenseNet169, DenseNet201, and DenseNet264. In this paper, we have used DenseNet201 for the pneumonia detection [51]. Each layer in DenseNet (as shown in Figure 5) has direct access to the original input image and gradients from the loss function. Therefore, the computational cost significantly reduced, which makes DenseNet a better choice for image classification.

\subsubsection{SqueezeNet}

SqueezeNet [52] is another CNN, which was trained using ImageNet database [51]. The SqueezeNet was trained with more than 1 million images and it has 50 times fewer parameters than AlexNet. The foundation of this network is a fire module, which consists of the squeeze layer and expand layer. The squeeze layer has only $1 \times 1$ filters, which are feeding to an expand layer that has a mixture of $1 \times 1$ and $3 \times 3$ convolution filters [52] (Figure 6). We have used the SqueezeNet pre-trained model to detect pneumonia and classify bacterial and viral pneumonia in this research. 


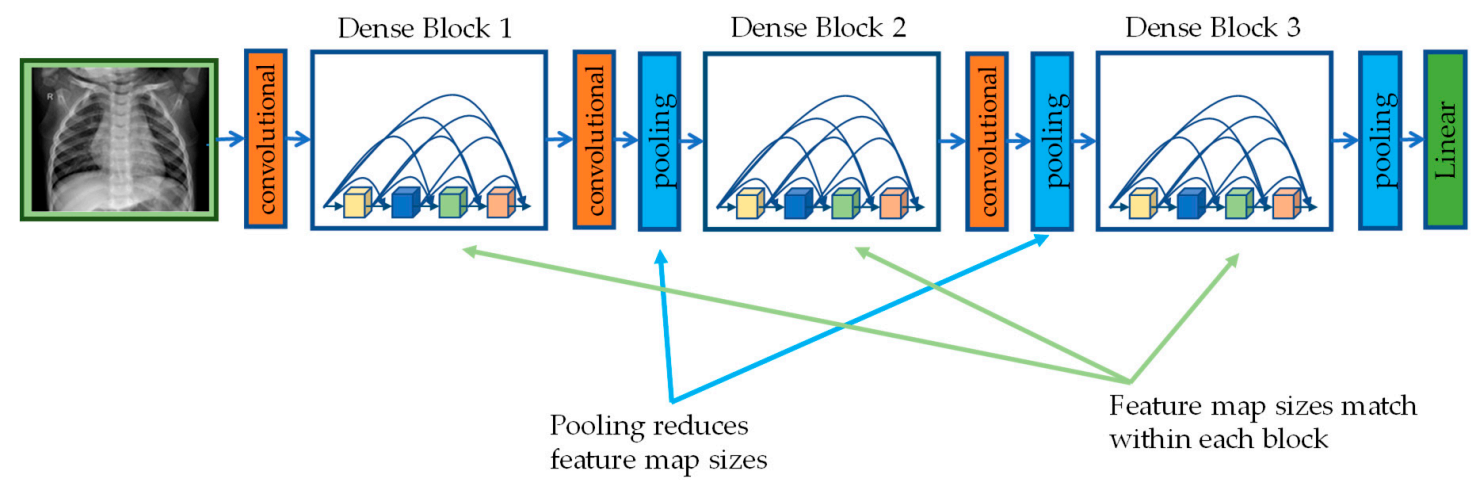

Figure 5. DenseNet201 architecture.
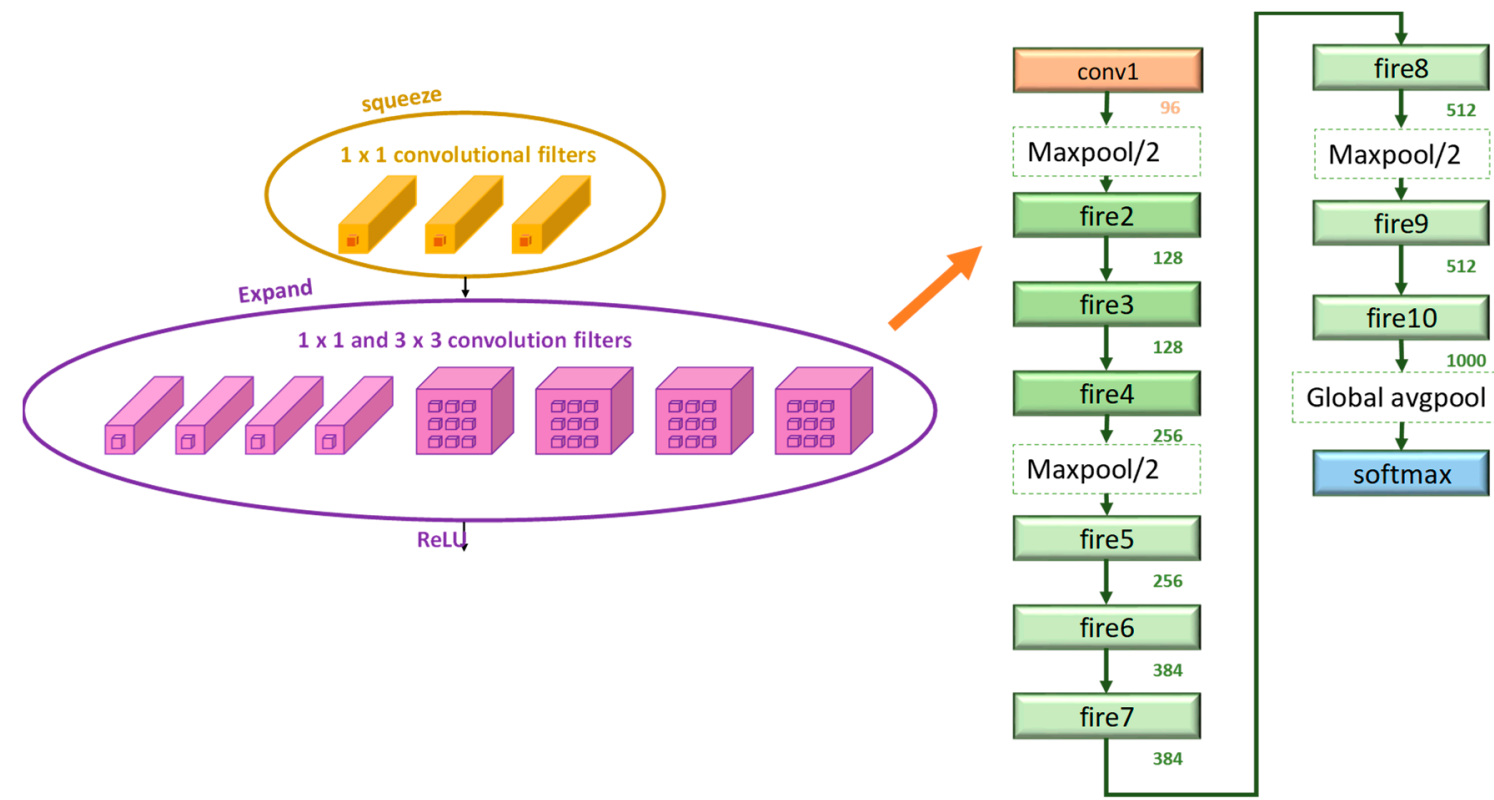

Figure 6. SqueezeNet architecture.

\section{Methodology}

\subsection{Dataset}

In this work, the kaggle chest $X$-ray pneumonia database was used, which is comprised of 5247 chest X-ray images with resolutions varying from 400p to 2000p [53]. Out of 5247 chest X-ray images, 3906 images are from different subjects affected by pneumonia (2561 images for bacterial pneumonia and 1345 images for viral pneumonia) and 1341 images are from normal subjects (Table 1). Mixed viral and bacterial infection occurs in some cases of pneumonia. However, the dataset used in this study does not include any case of viral and bacterial co-infection. This dataset was segmented into the training and test set.

Table 1. Complete dataset details.

\begin{tabular}{cc}
\hline Type & Number of X-ray Images \\
\hline Normal & 1341 \\
\hline Bacterial Pneumonia & 2561 \\
\hline Viral Pneumonia & 1345 \\
\hline Total & 5247 \\
\hline
\end{tabular}


Table 2 shows a number of train (using augmentation) and test images for different evaluation experiments. Four different algorithms were trained using the training dataset and then evaluated on test dataset. Figure 7 shows two samples for normal, bacterial, and viral pneumonia chest X-ray images.

Table 2. Details of the training and test set.

\begin{tabular}{|c|c|c|c|}
\hline \multicolumn{2}{|c|}{ Types } & $\begin{array}{c}\text { Training Set } \\
\text { (Using Augmentation) }\end{array}$ & Test Set \\
\hline \multirow{2}{*}{ Normal and Pneumonia } & Normal & 4500 & 205 \\
\hline & Pneumonia & 4500 & 214 \\
\hline \multirow{3}{*}{$\begin{array}{l}\text { Normal, Bacterial, } \\
\text { and Viral Pneumonia }\end{array}$} & Normal & 4500 & 199 \\
\hline & Bacterial Pneumonia & 4500 & 197 \\
\hline & Viral Pneumonia & 4500 & 201 \\
\hline \multirow{2}{*}{$\begin{array}{l}\text { Bacterial and Viral } \\
\text { Pneumonia }\end{array}$} & Bacterial Pneumonia & 4500 & 197 \\
\hline & Viral Pneumonia & 4500 & 201 \\
\hline
\end{tabular}

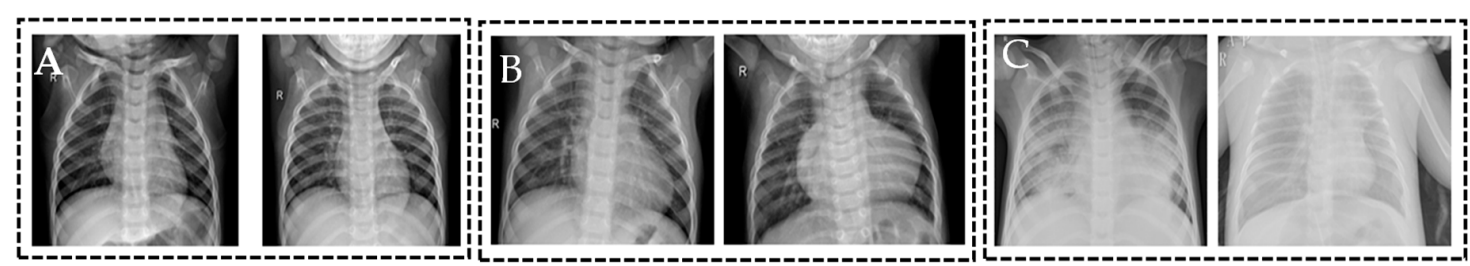

Figure 7. Data samples from the dataset, (A) shows normal cases, (B) shows bacterial pneumonia cases, and $(\mathbf{C})$ shows viral pneumonia cases.

In this study, MATLAB (2019a) was utilized to train, evaluate, and test different algorithms. Figure 8 illustrates the overview of the methodology of this study. Image sets undergo some pre-processing steps, data augmentation, and then training using pre-trained algorithms: AlexNet, ResNet18, DenseNet201, and SqueezeNet, and all the tested algorithms on the test dataset. The training of the different models was carried out in a computer with Intel@ i7-core @3.6GHz processor and 16GB RAM, 2 GB graphics card with graphics processing unit (GPU) on 64-bit Windows 10 operating system. Different parameters used for training the CNN models were shown in the Table 3. ResNet and DenseNet have different variants; however, ResNet18 and DenseNet201 were readily available for Matlab 2019a and therefore used in this study. Moreover, ResNet18 outperforms other ResNet models in terms of radiographic classification.

Table 3. Training parameters of different pre-trained CNN models.

\begin{tabular}{ccccccc}
\hline Software & $\begin{array}{c}\text { Pre-Trained } \\
\text { CNN Models }\end{array}$ & $\begin{array}{c}\text { Image } \\
\text { Size }\end{array}$ & Optimization & Momentum & Mini-Batch & Learning Rate \\
\cline { 2 - 3 } Matlab & AlexNet & $227 \times 227$ & & & \\
(2019a) & ResNet18 & $224 \times 224$ & Gradient & 0.9 & 16 & 0.0003 \\
\cline { 2 - 3 } & DenseNet201 & $224 \times 224$ & Descent & & & \\
\cline { 2 - 3 } & SqueezeNet & $227 \times 227$ & & & & \\
\hline
\end{tabular}




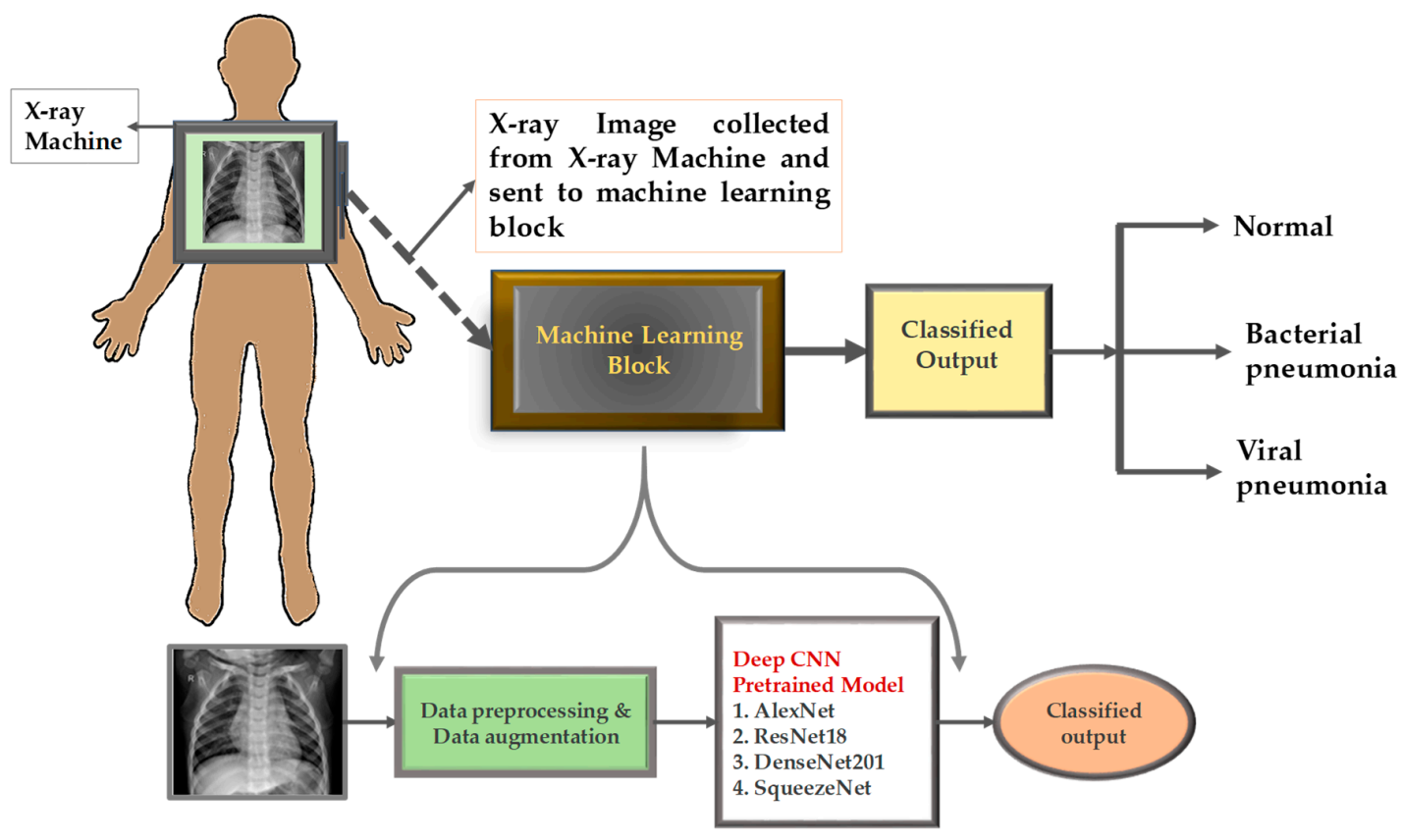

Figure 8. Overview of the methodology.

\subsection{Preprocessing}

One of the important steps in the data preprocessing was to resize the $\mathrm{X}$-ray images as the image input for different algorithms were different. For AlexNet and SqueezeNet, the images were resized to $227 \times 227$ pixels, whereas for ResNet18 and DenseNet201, the images were resized to and $224 \times 224$ pixels. All images were normalized according to the pre-trained model standards.

\section{Data Augmentation}

As discussed earlier, CNNs work better with a large dataset. However, the size of the working database is not very large. There is a common trend in training deep learning algorithms to make the comparatively smaller dataset a large one using data augmentation techniques. It is reported that data augmentation can improve the classification accuracy of the deep learning algorithms. The performance of the deep learning models can be improved by augmenting the existing data rather than collecting new data. Some of the deep learning frameworks have data augmentation facility built into the algorithms; however, in this study, authors have utilized three augmentation strategies to generate new training sets (Rotation, Scaling, and Translation), as shown in Figure 9.
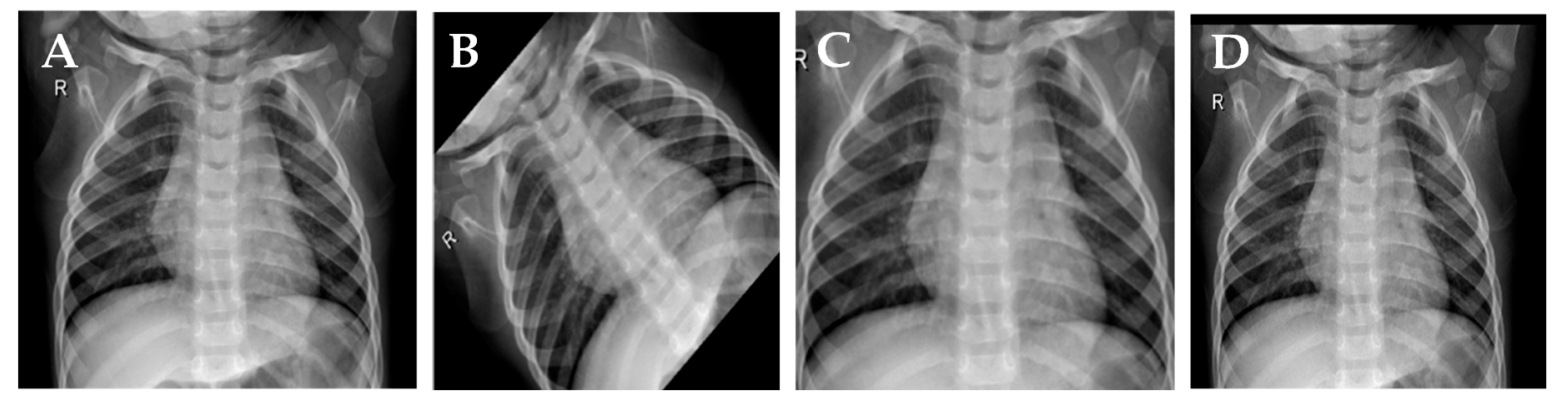

Figure 9. Original chest $X$-ray image (A), chest $X$-ray image after rotation $(\mathbf{B})$, chest $X$-ray image after scaling $(\mathbf{C})$, and chest-X-ray image after translation (D). 
The rotation operation used for image augmentation is normally done by rotating the image in the clockwise direction by an angle between 0 to 360 degrees, which rotates the pixel of the image frame and fills the area of the image where there was no image pixel. In this work, the rotation of 315 degrees (counter clockwise 45 degrees) was used. The scaling operation is the magnification or reduction of frame size of the image, which is another augmentation technique used. In total, $10 \%$ of image magnification, as done as shown in Figure 9C. Image translation can be done by either translating the image in a horizontal (width shift) or vertical direction (height shift) or in both directions. The original image was horizontally translated by $10 \%$ and vertically translated by $10 \%$.

\subsection{Visualization of the Activation Layer}

We investigated the features of the image by observing which areas in the convolutional layers activated on an image by comparing with the matching regions in the original images. Each layer of a CNN consists of many 2D arrays called channels. The input image was applied to different networks, and the output activations of the first convolution layer was examined.

The activations for different network models is shown in Figure 10. The activation map can take different range values and was therefore normalized between 0 and 1 . The strongest activation channels were observed and compared with the original image. It was noticed that this channel activates on edges. It activates positively on light left/dark right edges and negatively on dark left/light right edges.
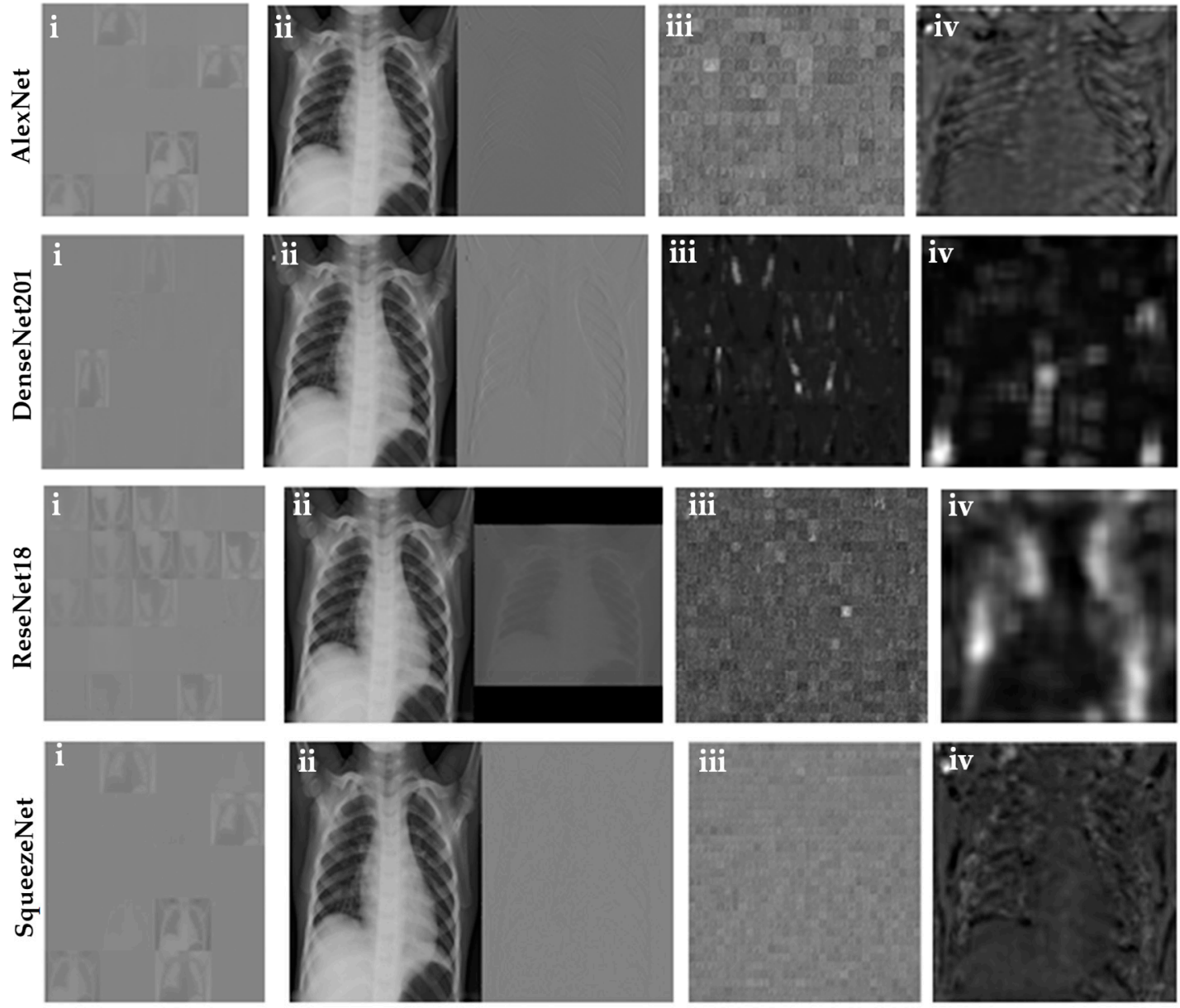

Figure 10. Activation map for different network models of (i) the first convolutional layer, (ii) the strongest activation channel, (iii) the deep layer images set, and (iv) the deep convolutional layer in specific image. 
Most convolutional neural networks learn to detect features like color and edges in their first convolutional layer. In deeper convolutional layers, the network learns to detect features that are more complicated. Later layers build up their features by combining features of earlier layers. Figure 10 shows the activation map in early convolutional layers, the deep convolutional layer, and the strongest activation channel for each of the models.

\subsection{Different Experiments}

Three different forms of performance evaluations and comparisons were carried out in this study: two classes (normal and pneumonia), three classes (normal, bacterial pneumonia, and viral pneumonia), and two classes (bacterial pneumonia and viral pneumonia) classification using four different deep learning algorithms through transfer learning.

The experiment carried out in this study consists of three steps. In the first step, the dataset divided into normal and pneumonia. In the second step, the dataset divided into normal, bacterial, and viral pneumonia. In the last step, the dataset divided into bacterial and viral pneumonia. An end-to-end training approach was adopted to classify normal, bacterial, and viral pneumonia images.

\subsection{Performance Matrix for Classification}

Four CNNs were trained and evaluated using five-fold cross-validation in this study. The performance of different networks for the testing dataset is evaluated after the completion of the training phase and was compared using the following six performances metrics: accuracy, sensitivity or recall, specificity, precision (PPV), the area under curve (AUC), and F1 score. Table 3 shows six performance metrics for different deep CNNs:

$$
\begin{gathered}
\text { Accuracy }=\frac{(T P+T N)}{(T P+F N)+(F P+T N)} \\
\text { Sensitivity }=\frac{(T P)}{(T P+F N)} \\
\text { Specificity }=\frac{(T N)}{(F P+T N)} \\
\text { Precision }=\frac{(T P)}{(T N+F P)} \\
\text { F1 Score }=\frac{(2 * T P)}{(2 * T P+F N+F P)}
\end{gathered}
$$

In the above equations, while classifying normal and pneumonia patients, true positive (TP), true negative (TN), false positive (FP), and false negative (FN) were used to denote the number of pneumonia images identified as pneumonia, the number of normal images identified as normal, the number normal images incorrectly identified as pneumonia images, and the number of pneumonia images incorrectly identified as normal, respectively. On the other hand, while classifying viral and bacterial pneumonia, TP, TN, FP, and FN were used to denote the number of viral pneumonia images identified as viral pneumonia, the number of bacterial pneumonia images identified as bacterial pneumonia, the number bacterial pneumonia images incorrectly identified as viral pneumonia images, and the number of viral pneumonia images incorrectly identified as bacterial pneumonia, respectively.

\section{Results and Discussions}

The comparative performance of training and testing accuracy for different CNNs for classification schemes are shown in Figure 11. It can be noted that, for three classification schemes, DenseNet201 is producing the highest accuracy for both training and testing. For normal and pneumonia classification, the test accuracy was $98 \%$, while, for normal, bacterial, and viral pneumonia classification, it was 
$93.3 \%$, and, for bacterial and viral pneumonia classification, it was found to be $95 \%$. Figure 12 shows the area under the curve (AUC)/receiver-operating characteristics (ROC) curve (also known as AUROC (area under the receiver operating characteristics)) for different classification schemes, which is one of the most important evaluation metrics for checking any classification model's performance. It is also evident from Figure 12 that DenseNet201 outperforms the other algorithms.
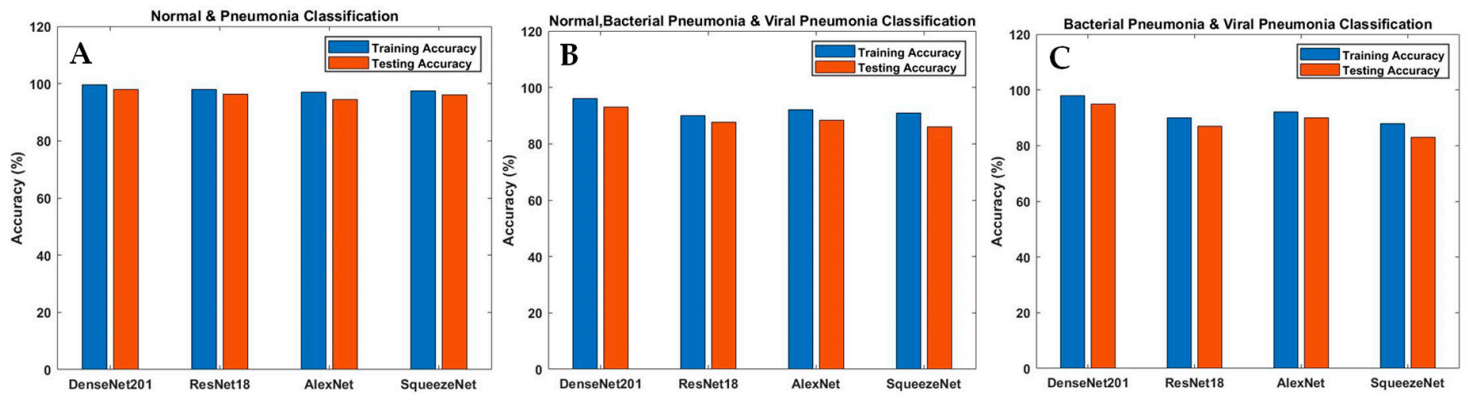

Figure 11. Comparison of training and testing accuracy for normal pneumonia (A), normal, bacterial, and viral pneumonia (B), and bacterial and viral pneumonia (C) classification using different models.
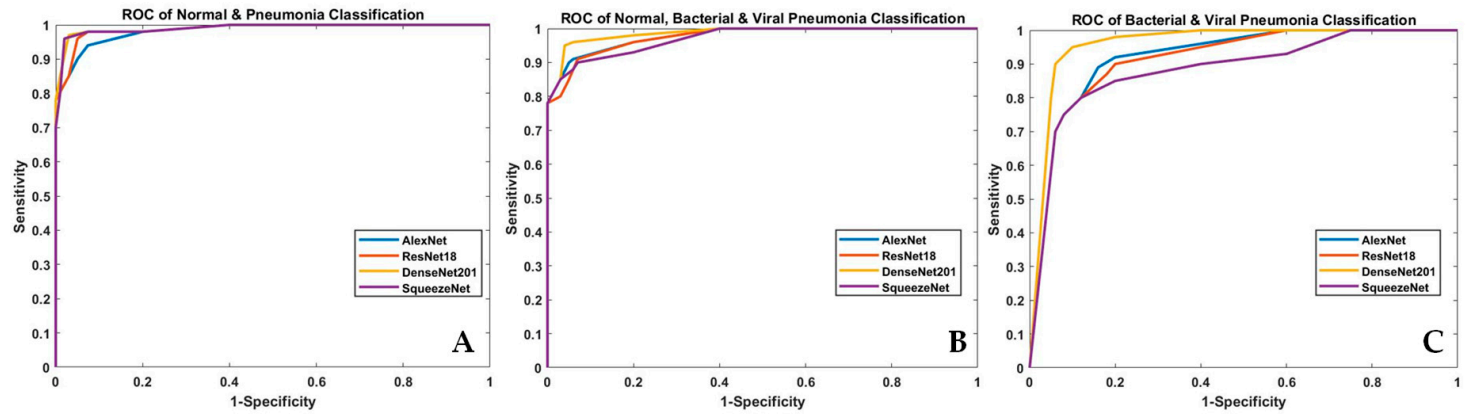

Figure 12. Comparison of the receiver-operating characteristics (ROC) curve for normal pneumonia (A), normal, bacterial, and viral pneumonia (B), and bacterial and viral pneumonia (C) classification using CNN-based models.

Figure 13 shows the confusion matrix for outperforming Densenet201 pre-trained model in for three different classifications.

Table 4 summarizes the performance matrix for different $\mathrm{CNN}$ algorithms tested for the three different classification schemes. DenseNet201 outperforms other models in three different classification schemes in terms of different performance indices.

The authors have also compared the results in the paper with the results of recently published works in the same problem. Vikash et al. [21] reported the detection of pneumonia using a deep learning technique called CXNet-m1, which showed sensitivity, precision, and accuracy of $99.6 \%$, $93.28 \%$, and $96.39 \%$, respectively. Krishnan et al. [29] evaluated the performance of VGG16 CNNs to classify pneumonia into normal, bacterial, and viral pneumonias. This showed sensitivity, precision, and accuracy of $99.5 \%, 97.7 \%$, and $96.2 \%$, respectively for pneumonia detection; however, $98.4 \%, 92 \%$, and $93.6 \%$, respectively, for distinguishing viral and bacterial pneumonias. Table 5 summarized the comparison of others works in pneumonia and types of pneumonia detection from chest $\mathrm{X}$-ray images. 


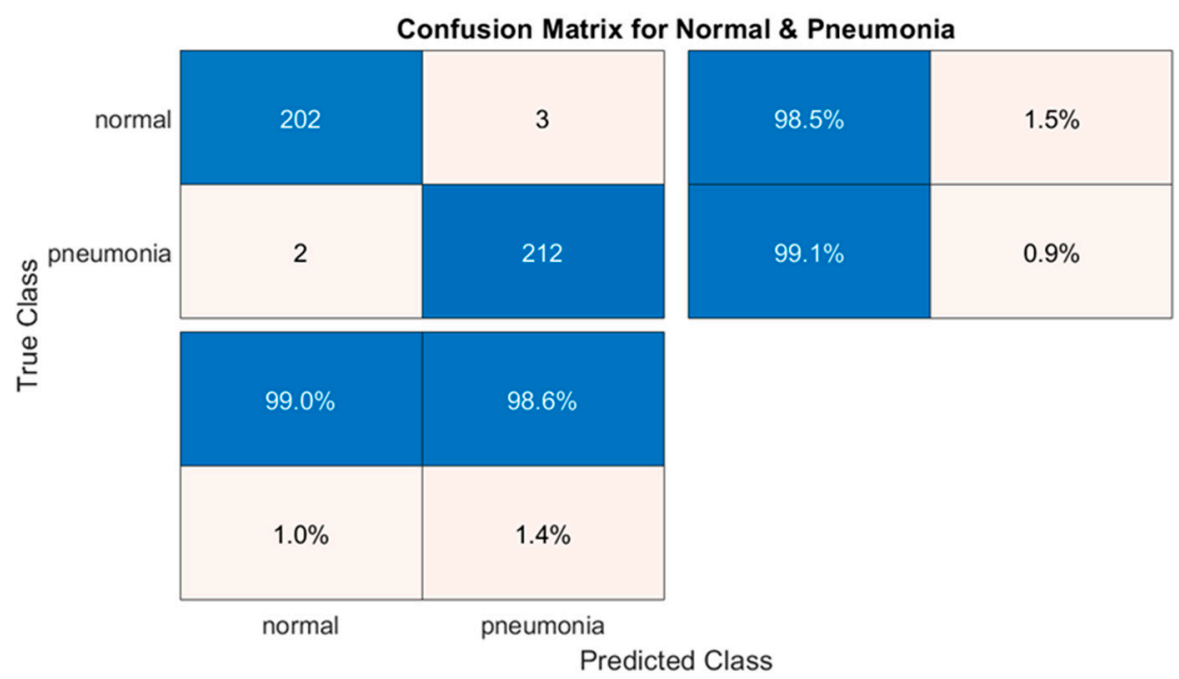

(a)

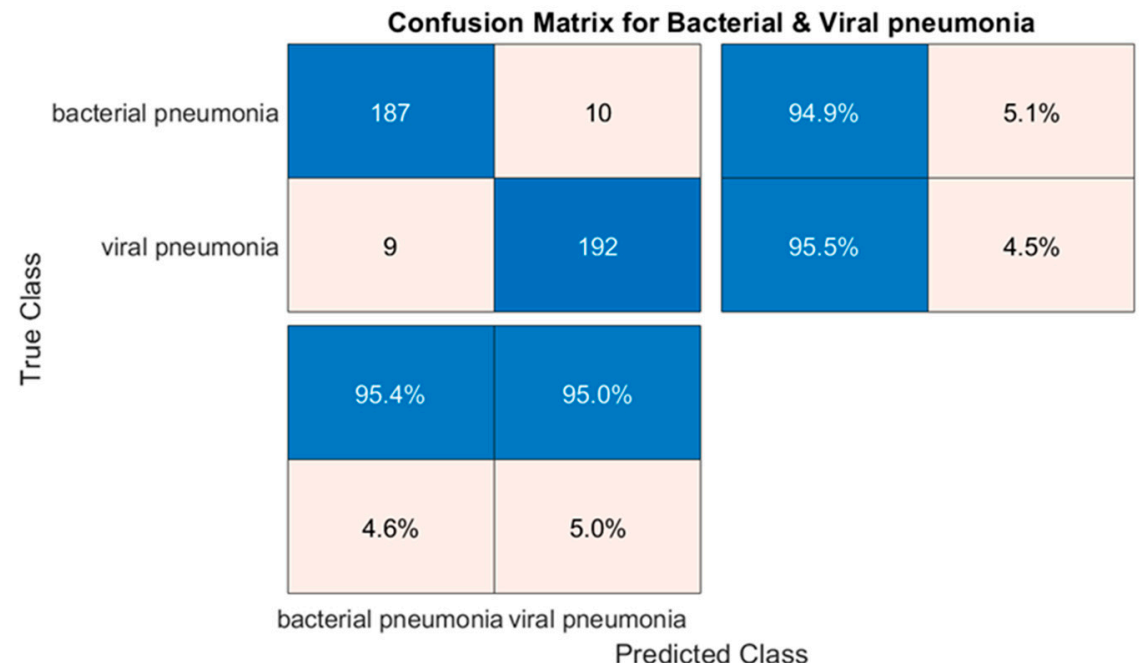

(b)

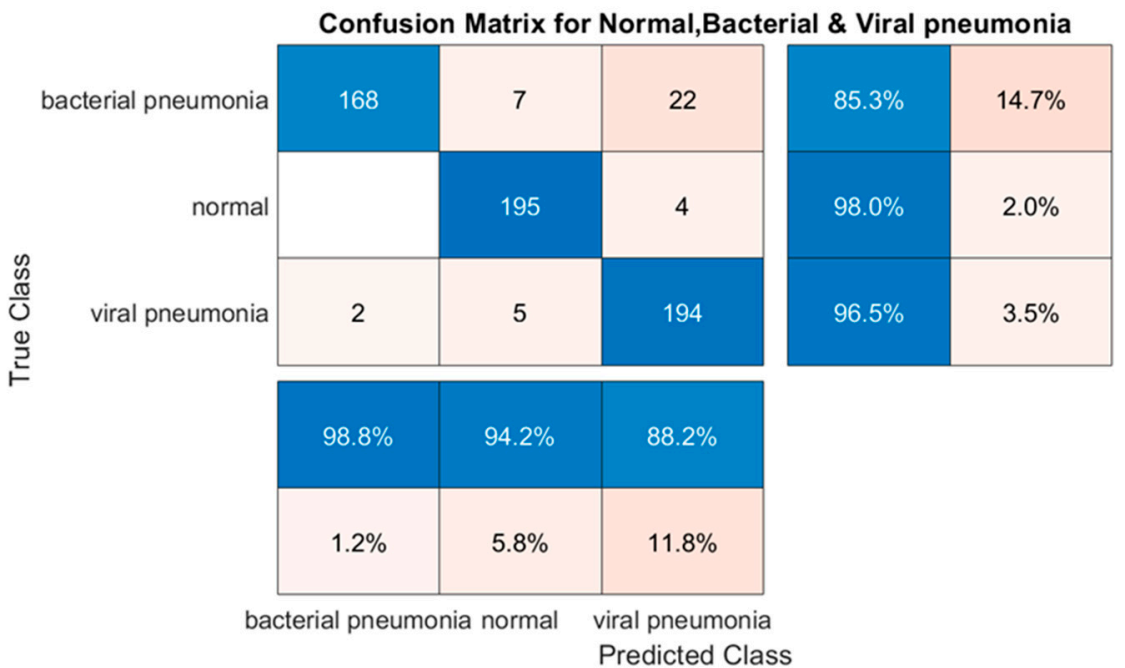

(c)

Figure 13. Confusion matrix for (a) normal pneumonia, (b) bacterial and viral, and (c) normal, bacterial, and viral pneumonia classification using DenseNet201. 
Table 4. Different performance metrics for different deep learning networks.

\begin{tabular}{cccccccc}
\hline Task & Models & Accuracy & $\begin{array}{c}\text { Sensitivity } \\
\text { (Recall) }\end{array}$ & Specificity & $\begin{array}{c}\text { Precision } \\
\text { (PPV) }\end{array}$ & $\begin{array}{c}\text { Area under } \\
\text { Curve (AUC) }\end{array}$ & F1 Scores \\
\hline \multirow{3}{*}{$\begin{array}{c}\text { Normal and } \\
\text { Pneumonia }\end{array}$} & AlexNet & 0.945 & 0.953 & 0.926 & 0.931 & 0.942 & 0.943 \\
\cline { 2 - 8 } & ResNet18 & 0.964 & 0.97 & 0.95 & 0.954 & 0.963 & 0.965 \\
\cline { 2 - 8 } & DenseNet201 & $\mathbf{0 . 9 8}$ & 0.99 & 0.97 & 0.97 & 0.98 & 0.981 \\
\cline { 2 - 8 } & SqueezeNet & 0.961 & 0.94 & 0.98 & 0.985 & 0.96 & 0.961 \\
\hline \multirow{2}{*}{$\begin{array}{c}\text { Normal, Bacterial } \\
\text { Pneumonia and }\end{array}$} & AlexNet & 0.884 & 0.883 & 0.941 & 0.886 & 0.911 & 0.885 \\
\cline { 2 - 8 } Viral Pneumonia & ResNet18 & 0.877 & 0.88 & 0.94 & 0.875 & 0.91 & 0.909 \\
\cline { 2 - 8 } & SqueNet201 & $\mathbf{0 . 9 3 3}$ & 0.932 & 0.967 & 0.937 & 0.95 & 0.935 \\
\hline \multirow{3}{*}{$\begin{array}{c}\text { Bacterial and Viral } \\
\text { Pneumonia }\end{array}$} & AlexNet & 0.861 & 0.959 & 0.93 & 0.87 & 0.895 & 0.865 \\
\cline { 2 - 8 } & ResNet18 & 0.87 & 0.94 & 0.845 & 0.86 & 0.89 & 0.921 \\
\cline { 2 - 8 } & DenseNet201 & $\mathbf{0 . 9 5}$ & 0.96 & 0.94 & 0.83 & 0.87 & 0.873 \\
\cline { 2 - 8 } & SqueezeNet & 0.83 & 0.905 & 0.75 & 0.79 & 0.952 & 0.952 \\
\hline
\end{tabular}

Table 5. Comparison with other recent similar works.

\begin{tabular}{|c|c|c|c|c|c|c|c|}
\hline Author & Classes & Technique & $\begin{array}{c}\text { Image } \\
\text { No. }\end{array}$ & $\begin{array}{c}\text { Recall } \\
\text { /Sensitivity(\%) }\end{array}$ & $\begin{array}{l}\text { Precision } \\
(\%)\end{array}$ & $\begin{array}{c}\text { AUC } \\
(\%)\end{array}$ & $\begin{array}{c}\text { Accuracy } \\
(\%)\end{array}$ \\
\hline Vikash et al. [21] & $\begin{array}{l}\text { Normal \& } \\
\text { Pneumonia }\end{array}$ & $\begin{array}{c}\text { Different } \\
\text { pre-trained CNN } \\
\text { model }\end{array}$ & 5232 & 99.6 & 93.28 & 99.34 & 96.39 \\
\hline $\begin{array}{c}\text { Sivarama krishnan } \\
\text { et al. [29] }\end{array}$ & $\begin{array}{c}\text { Normal \& } \\
\text { Pneumonia }\end{array}$ & $\begin{array}{c}\text { customized } \\
\text { VGG16 CNN model }\end{array}$ & 5856 & 99.5 & 97.0 & 99.0 & 96.2 \\
\hline Kermanyetal. [54] & $\begin{array}{l}\text { Normal \& } \\
\text { Pneumonia }\end{array}$ & $\begin{array}{c}\text { Inception } \\
\text { V3 pretrained CNN } \\
\text { model }\end{array}$ & 5232 & 93.2 & 90.1 & - & 92.8 \\
\hline $\begin{array}{l}{ }^{*} \text { M.Togacar } \\
\text { et al. [22] }\end{array}$ & $\begin{array}{c}\text { Normal \& } \\
\text { Pneumonia }\end{array}$ & Deep CNN model & 5849 & 96.83 & 96.88 & 96.80 & 96.84 \\
\hline $\begin{array}{l}\text { A. A. Saraiva } \\
\text { et al. [23] }\end{array}$ & $\begin{array}{c}\text { Normal \& } \\
\text { Pneumonia }\end{array}$ & Neural network & 5840 & 94.5 & 94.3 & 94.5 & 94.4 \\
\hline $\begin{array}{l}\text { Enes AYAN } \\
\text { et al. [24] }\end{array}$ & $\begin{array}{c}\text { Normal \& } \\
\text { Pneumonia }\end{array}$ & $\begin{array}{c}\text { VGG16 deep } \\
\text { learning model }\end{array}$ & 5856 & 89.1 & 91.3 & 87.0 & 84.5 \\
\hline $\begin{array}{l}\text { Xianghong Gu } \\
\text { et al. [30] }\end{array}$ & $\begin{array}{c}\text { Bacterial \& } \\
\text { Viral } \\
\text { Pneumonia }\end{array}$ & $\begin{array}{l}\text { Deep Convolutional } \\
\text { neural network }\end{array}$ & 4882 & 77.55 & 88.86 & 82.3 & 80.4 \\
\hline $\begin{array}{l}\text { * Sivarama krishnan } \\
\text { et al. [29] }\end{array}$ & $\begin{array}{c}\text { Bacterial \& } \\
\text { Viral } \\
\text { Pneumonia }\end{array}$ & $\begin{array}{c}\text { customized } \\
\text { VGG16 CNN model }\end{array}$ & 3883 & 98.4 & 92.0 & 96.2 & 93.6 \\
\hline $\begin{array}{l}\text { Archit Khatri } \\
\text { et al. [25] }\end{array}$ & $\begin{array}{c}\text { Bacterial \& } \\
\text { Viral } \\
\text { Pneumonia }\end{array}$ & EMD approach & 144 & 89.5 & 80.0 & 88.0 & 83.30 \\
\hline${ }^{* *}$ This work & $\begin{array}{c}\text { Normal \& } \\
\text { Pneumonia }\end{array}$ & $\begin{array}{c}\text { Different } \\
\text { pre-trained CNN } \\
\text { model }\end{array}$ & 5247 & 99.0 & 97.0 & 98.0 & 98.0 \\
\hline${ }^{* *}$ This work & $\begin{array}{c}\text { Bacterial \& } \\
\text { Viral } \\
\text { Pneumonia }\end{array}$ & $\begin{array}{c}\text { Different } \\
\text { pre-trained CNN } \\
\text { model }\end{array}$ & 5247 & 96.0 & 95.0 & 95.2 & 95.0 \\
\hline
\end{tabular}

* The best performing algorithms reported in the literature while ** shows the performance of this study.

It is evident that DesneNet201 exhibits the highest accuracy of all recent studies to the best of our knowledge, which could be useful in developing a prototype that can automatically classify results into normal, bacterial and viral pneumonia. Training the network using a larger database and working on an ensemble of the pre-trained $\mathrm{CNN}$ algorithms might increase the detection accuracy, which can be done as a future study.

\section{Conclusions}

This study presents a deep-CNN-based transfer learning approach for the automatic detection of pneumonia and its classes. Four different popular CNN-based deep learning algorithms were trained and tested for classifying normal and pneumonia patients using chest x-ray images. It was observed that DenseNet201 outperforms the other three different deep CNN networks. The classification 
accuracy, precision, and recall of normal and pneumonia images, bacterial, and viral pneumonia images, and normal, bacterial, and viral pneumonia were $98 \%, 97 \%$, and $99 \% ; 95 \%, 95 \%$ and $96 \%$; and $93.3 \%, 93.7 \%$ and $93.2 \%$, respectively. There are millions of children, who die each year due to this potentially fatal disease. Timely intervention with proper treatment plan through correct diagnosis of the disease can save a significant number of lives. Due to the huge number of patients in outdoor emergencies or emergencies specifically in the third world countries, medical doctors' time is limited and computer-aided-diagnosis (CAD) can save lives. Moreover, there is a large degree of variability in the input images from the X-ray machines due to the variations in the expertise of the radiologists. DenseNet201 exhibits an excellent performance in classifying pneumonia by effectively training itself from a comparatively lower collection of complex data, such as images, with reduced bias and higher generalization. We believe that this computer-aided diagnostic tool can significantly help the radiologist to take more clinically useful images and to identify pneumonia with its type immediately after acquisition. This fast classification will open up other avenues of application for this CAD tool, more particularly in the airport screening of pneumonia patients.

Author Contributions: Experiments were designed by T.R. and M.E.H.C. Experiments were performed by T.R., K.F.I. and K.R.I. Results were analyzed by T.R., M.E.H.C., A.K., S.B., Z.B.M. and M.A.K. All authors were involved in interpretation of data and paper writing. All authors have read and agreed to the published version of the manuscript.

Funding: This work was made possible by NPRP12S-0227-190164 from the Qatar National Research Fund, a member of Qatar Foundation, Doha, Qatar and students' grant of Qatar University QUST-1-CENG-2020-23. The statements made herein are solely the responsibility of the authors.

Conflicts of Interest: The authors declare no conflict of interest.

\section{References}

1. Imran, A. Training a CNN to detect Pneumonia. 2019. Available online: https://medium.com/ datadriveninvestor/training-a-cnn-to-detect-pneumonia-c42a44101deb (accessed on 23 December 2019).

2. Aydogdu, M.; Ozyilmaz, E.; Aksoy, H.; Gursel, G.; Ekim, N. Mortality prediction in community-acquired pneumonia requiring mechanical ventilation; values of pneumonia and intensive care unit severity scores. Tuberk Toraks 2010, 58, 25-34. [PubMed]

3. WHO. Standardization of Interpretation of Chest Radiographs for the Diagnosis of Pneumonia in Children; World Health Organization: Geneva, Switzerland, 2001.

4. Neuman, M.I.; Lee, E.Y.; Bixby, S.; DiPerna, S.; Hellinger, J.; Markowitz, R.; Servaes, S.; Monuteaux, M.C.; Shah, S. Variability in the interpretation of chest radiographs for the diagnosis of pneumonia in children. J. Hosp. Med. 2011, 7, 294-298. [CrossRef] [PubMed]

5. Dele Davies, H.; Wang, E.E.-L.; Manson, D.; Babyn, P.S.; Shuckett, B. Reliability of the chest radiograph in the diagnosis of lower respiratory infections in young children. Pediatr. Infect. Dis. J. 1996, 15, 600-604. [CrossRef] [PubMed]

6. Hopstaken, R.; Witbraad, T.; Van Engelshoven, J.; Dinant, G. Inter-observer variation in the interpretation of chest radiographs for pneumonia in community-acquired lower respiratory tract infections. Clin. Radiol. 2004, 59, 743-752. [CrossRef] [PubMed]

7. Tahir, A.M.; Chowdhury, M.E.H.; Khandakar, A.; Al-Hamouz, S.; Abdalla, M.; Awadallah, S.; Reaz, M.B.I.; Al-Emadi, N. A Systematic Approach to the Design and Characterization of a Smart Insole for Detecting Vertical Ground Reaction Force (vGRF) in Gait Analysis. Sensors 2020, 20, 957. [CrossRef]

8. Chowdhury, M.E.H.; Alzoubi, K.; Khandakar, A.; Khallifa, R.; Abouhasera, R.; Koubaa, S.; Ahmed, R.; Hasan, A. Wearable Real-Time Heart Attack Detection and Warning System to Reduce Road Accidents. Sensors 2019, 19, 2780. [CrossRef]

9. Chowdhury, M.E.H.; Khandakar, A.; Alzoubi, K.; Mansoor, S.; Tahir, A.M.; Reaz, M.B.I.; Al-Emadi, N. Real-Time Smart-Digital Stethoscope System for Heart Diseases Monitoring. Sensors 2019, 19, 2781. [CrossRef]

10. Kallianos, K.; Mongan, J.; Antani, S.; Henry, T.; Taylor, A.; Abuya, J.; Kohli, M. How far have we come? Artificial intelligence for chest radiograph interpretation. Clin. Radiol. 2019, 74, 338-345. [CrossRef] 
11. Krizhevsky, A.; Sutskever, I.; Hinton, G.E. Pdf ImageNet classification with deep convolutional neural networks. Commun. ACM 2017, 60, 84-90. [CrossRef]

12. Wang, X.; Peng, Y.; Lu, L.; Lu, Z.; Bagheri, M.; Summers, R.M. ChestX-ray8: Hospital-Scale Chest X-ray Database and Benchmarks on Weakly-Supervised Classification and Localization of Common Thorax Diseases. In Proceedings of the 2017 IEEE Conference on Computer Vision and Pattern Recognition (CVPR), Honolulu, HI, USA, 21-26 July 2017; pp. 3462-3471.

13. Ronneberger, O.; Fischer, P.; Brox, T. U-Net: Convolutional Networks for Biomedical Image Segmentation. In Proceedings of the Lecture Notes in Computer Science; Springer Science and Business Media LLC: Cham, Switzerland, 2015; Volume 9351, pp. 234-241.

14. Woźniak, M.; Polap, D.; Capizzi, G.; Sciuto, G.L.; Kośmider, L.; Frankiewicz, K. Small lung nodules detection based on local variance analysis and probabilistic neural network. Comput. Methods Programs Biomed. 2018, 161, 173-180. [CrossRef]

15. Gu, Y.; Lu, X.; Yang, L.; Zhang, B.; Yu, D.; Zhao, Y.; Gao, L.; Wu, L.; Zhou, T. Automatic lung nodule detection using a 3D deep convolutional neural network combined with a multi-scale prediction strategy in chest CTs. Comput. Boil. Med. 2018, 103, 220-231. [CrossRef] [PubMed]

16. Ho, T.K.K.; Gwak, J. Multiple Feature Integration for Classification of Thoracic Disease in Chest Radiography. Appl. Sci. 2019, 9, 4130. [CrossRef]

17. Jaiswal, A.K.; Tiwari, P.; Kumar, S.; Gupta, N.; Khanna, A.; Rodrigues, J.J.P.C. Identifying pneumonia in chest X-rays: A deep learning approach. Measurement 2019, 145, 511-518. [CrossRef]

18. Liang, G.; Zheng, L. A transfer learning method with deep residual network for pediatric pneumonia diagnosis. Comput. Methods Programs Biomed. 2020, 187, 104964. [CrossRef]

19. Souza, J.C.; Diniz, J.O.B.; Ferreira, J.L.; Da Silva, G.L.F.; Silva, A.C.; De Paiva, A.C. An automatic method for lung segmentation and reconstruction in chest X-ray using deep neural networks. Comput. Methods Programs Biomed. 2019, 177, 285-296. [CrossRef]

20. Taylor, A.G.; Mielke, C.; Mongan, J. Automated detection of moderate and large pneumothorax on frontal chest X-rays using deep convolutional neural networks: A retrospective study. PLoS Med. 2018, 15, e1002697. [CrossRef]

21. Xu, S.; Wu, H.; Bie, R. CXNet-m1: Anomaly Detection on Chest X-rays With Image-Based Deep Learning. IEEE Access 2018, 7, 4466-4477. [CrossRef]

22. Toğaçar, M.; Ergen, B.; Cömert, Z.; Özyurt, F. A Deep Feature Learning Model for Pneumonia Detection Applying a Combination of mRMR Feature Selection and Machine Learning Models. IRBM 2019. [CrossRef]

23. Saraiva, A.; Santos, D.; Costa, N.; Sousa, J.; Ferreira, N.; Valente, A.; Soares, S. Models of Learning to Classify X-ray Images for the Detection of Pneumonia using Neural Networks. In Proceedings of the 12th International Joint Conference on Biomedical Engineering Systems and Technologies, Prague, Czech, 22-24 February 2019; pp. 76-83.

24. Ayan, E.; Unver, H.M. Diagnosis of Pneumonia from Chest X-ray Images Using Deep Learning. In Proceedings of the 2019 Scientific Meeting on Electrical-Electronics \& Biomedical Engineering and Computer Science (EBBT), Istanbul, Turkey, 24-26 April 2019; pp. 1-5.

25. Khatri, A.; Jain, R.; Vashista, H.; Mittal, N.; Ranjan, P.; Janardhanan, R. Pneumonia Identification in Chest X-ray Images Using EMD. In Internet of Things-Applications and Future; Springer Science and Business Media LLC: Singapore, 2020; pp. 87-98.

26. Virkki, R.; Juvén, T.; Rikalainen, H.; Svedstrom, E.; Mertsola, J.; Ruuskanen, O. Differentiation of bacterial and viral pneumonia in children. Thorax 2002, 57, 438-441. [CrossRef]

27. Ishiguro, T.; Kobayashi, Y.; Uozumi, R.; Takata, N.; Takaku, Y.; Kagiyama, N.; Kanauchi, T.; Shimizu, Y.; Takayanagi, N. Viral Pneumonia Requiring Differentiation from Acute and Progressive Diffuse Interstitial Lung Diseases. Intern. Med. 2019, 58, 3509-3519. [CrossRef]

28. Chouhan, V.; Singh, S.K.; Khamparia, A.; Gupta, N.; Tiwari, P.; Moreira, C.; Damasevicius, R.; De Albuquerque, V.H.C. A Novel Transfer Learning Based Approach for Pneumonia Detection in Chest X-ray Images. Appl. Sci. 2020, 10, 559. [CrossRef]

29. Rajaraman, S.; Candemir, S.; Kim, I.; Thoma, G.R.; Antani, S. Visualization and Interpretation of Convolutional Neural Network Predictions in Detecting Pneumonia in Pediatric Chest Radiographs. Appl. Sci. 2018, 8, 1715. [CrossRef] 
30. Gu, X.; Pan, L.; Liang, H.; Yang, R. Classification of Bacterial and Viral Childhood Pneumonia Using Deep Learning in Chest Radiography. In Proceedings of the 3rd international conference on Mobile and ubiquitous multimedia-MUM '04; Association for Computing Machinery (ACM), Guiyang China, 16-18 March 2018; pp. 88-93.

31. Gershgorn, D. The Data that Transformed AI Research—and Possibly the World. 2017. Available online: https: //qz.com/1034972/the-data-that-changed-the-direction-of-ai-research-and-possibly-the-world/ (accessed on 23 December 2019).

32. Rajpurkar, P.; Irvin, J.; Ball, R.L.; Zhu, K.; Yang, B.; Mehta, H.; Duan, T.; Ding, D.; Bagul, A.; Langlotz, C.P.; et al. Deep learning for chest radiograph diagnosis: A retrospective comparison of the CheXNeXt algorithm to practicing radiologists. PLoS Med. 2018, 15, e1002686. [CrossRef] [PubMed]

33. Jung, H.; Kim, B.; Lee, I.; Lee, J.; Kang, J. Classification of lung nodules in CT scans using three-dimensional deep convolutional neural networks with a checkpoint ensemble method. BMC Med. Imag. 2018, 18, 48. [CrossRef] [PubMed]

34. Sirazitdinov, I.; Kholiavchenko, M.; Mustafaev, T.; Yixuan, Y.; Kuleev, R.; Ibragimov, B. Deep neural network ensemble for pneumonia localization from a large-scale chest x-ray database. Comput. Electr. Eng. 2019, 78, 388-399. [CrossRef]

35. Lakhani, P.; Sundaram, B. Deep Learning at Chest Radiography: Automated Classification of Pulmonary Tuberculosis by Using Convolutional Neural Networks. Radiology 2017, 284, 574-582. [CrossRef]

36. Szegedy, C.; Liu, W.; Jia, Y.; Sermanet, P.; Reed, S.; Anguelov, D.; Erhan, D.; Vanhoucke, V.; Rabinovich, A. Going deeper with convolutions. In Proceedings of the 2015 IEEE Conference on Computer Vision and Pattern Recognition (CVPR), Boston, MA, USA, 7-12 June 2015; pp. 1-9.

37. Albawi, S.; Mohammed, T.A.; Al-Azawi, S. Understanding of a convolutional neural network. In Proceedings of the 2017 International Conference on Engineering and Technology (ICET), Antalya, Turkey, 21-23 August 2017; pp. 1-6.

38. Goyal, M.; Goyal, R.; Lall, B. Learning Activation Functions: A new paradigm of understanding Neural Networks. arXiv 2019, arXiv:1906.09529.

39. Bailer, C.; Habtegebrial, T.; Varanasi, K.; Stricker, D. Fast Feature Extraction with CNNs with Pooling Layers. arXiv 2018, arXiv:1805.03096.

40. Deng, J.; Dong, W.; Socher, R.; Li, L.-J.; Li, K.; Fei-Fei, L. Imagenet: A large-scale hierarchical image database. In Proceedings of the 2009 IEEE Conference on Computer Vision and Pattern Recognition, Miami, FL, USA, 20-25 June 2009; pp. 248-255.

41. Wang, S.-H.; Xie, S.; Chen, X.; Guttery, D.; Tang, C.; Sun, J.; Zhang, Y.-D. Alcoholism Identification Based on an AlexNet Transfer Learning Model. Front. Psychol. 2019, 10, 205. [CrossRef]

42. Christodoulidis, S.; Anthimopoulos, M.; Ebner, L.; Christe, A.; Mougiakakou, S.G. Multisource Transfer Learning With Convolutional Neural Networks for Lung Pattern Analysis. IEEE J. Biomed. Health Inform. 2016, 21, 76-84. [CrossRef]

43. Yang, H.; Mei, S.; Song, K.; Tao, B.; Yin, Z. Transfer-Learning-Based Online Mura Defect Classification. IEEE Trans. Semicond. Manuf. 2017, 31, 116-123. [CrossRef]

44. Akcay, S.; Kundegorski, M.E.; Devereux, M.; Breckon, T.P. Transfer learning using convolutional neural networks for object classification within X-ray baggage security imagery. In Proceedings of the 2016 IEEE International Conference on Image Processing (ICIP), Phoenix, AZ, USA, 25-28 September 2016; pp. 1057-1061.

45. Tajbakhsh, N.; Shin, J.Y.; Gurudu, S.R.; Hurst, R.T.; Kendall, C.B.; Gotway, M.B.; Liang, J. Convolutional Neural Networks for Medical Image Analysis: Full Training or Fine Tuning? IEEE Trans. Med. Imag. 2016, 35, 1299-1312. [CrossRef] [PubMed]

46. Pan, S.J.; Yang, Q. A Survey on Transfer Learning. IEEE Trans. Knowl. Data Eng. 2009, 22, 1345-1359. [CrossRef]

47. ResNet, AlexNet, VGGNet, Inception: Understanding various architectures of Convolutional Networks. Available online: https://cv-tricks.com/cnn/understand-resnet-alexnet-vgg-inception/ (accessed on 23 December 2019).

48. LeCun, Y.; Kavukcuoglu, K.; Farabet, C. Convolutional networks and applications in vision. In Proceedings of the 2010 IEEE International Symposium on Circuits and Systems, Paris, France, 30 May-2 June 2010; pp. 253-256. 
49. LeCun, Y.; Boser, B.E.; Denker, J.S.; Henderson, D.; Howard, R.E.; Hubbard, W.E.; Jackel, L.D. Handwritten digit recognition with a back-propagation network. In Advances in Neural Information Processing Systems; The MIT Press: Massachusetts, MA, USA, 1990; pp. 396-404.

50. Huang, G.; Liu, Z.; Van Der Maaten, L.; Weinberger, K.Q. Densely Connected Convolutional Networks. In Proceedings of the 2017 IEEE Conference on Computer Vision and Pattern Recognition (CVPR), Honolulu, HI, USA, 21-26 July 2017; pp. 2261-2269.

51. DenseNet: Better CNN Model than ResNet. Available online: http://www.programmersought.com/article/ 7780717554/ (accessed on 23 December 2019).

52. Iandola, F.N.; Han, S.; Moskewicz, M.W.; Ashraf, K.; Dally, W.J.; Keutzer, K. SqueezeNet: AlexNet-level accuracy with 50x fewer parameters and <0.5 MB model size. arXiv 2016, arXiv:1602.07360.

53. Mooney, P. Chest X-ray Images (Pneumonia). 2018. Available online: https://www.kaggle.com/ paultimothymooney/chest-xray-pneumonia (accessed on 23 December 2019).

54. Cohen, J.P.; Bertin, P.; Frappier, V. Chester: A Web Delivered Locally Computed Chest X-ray Disease Prediction System. arXiv 2019, arXiv:1901.11210.

(C) 2020 by the authors. Licensee MDPI, Basel, Switzerland. This article is an open access article distributed under the terms and conditions of the Creative Commons Attribution (CC BY) license (http://creativecommons.org/licenses/by/4.0/). 